\title{
" TRAYECTORIAS INTELECTUALES, PENSAMIENTO Y MODOS DE INTERVENCIÓN PÚBLICA DE AGENTES UNIVERSITARIOS ARGENTINOS EN EL SIGLO XX "1
}

El marco de reflexión y debate generado por el centenario de la Reforma Universitaria, en junio de 2018, dio lugar a la reciente publicación de obras colectivas, acervos documentales y balances historiográficos. ${ }^{2}$ Estas producciones respondían a la vez a la renovación y pluralidad de enfoques que se ha operado en las últimas décadas en los estudios sobre el tema. De la mano de la historia intelectual, la renovación de la historia política, la historia social y cultural, estos trabajos contribuyeron, entre otros aspectos, a reponer la complejidad de posiciones e identidades a las que dio lugar el reformismo como así también a reconstruir las redes y derivas locales y transnacionales en las que se articularon los reclamos gremiales, corporativos y educativos de la Reforma y aquellos que incluían una proyección extra universitaria de ese movimiento.

En este contexto, los trabajos reunidos en el presente dossier aportan una reconstrucción de las modalidades de intervención pública de diversos agentes universitarios (estudiantes, intelectuales, asociaciones, agrupaciones, revistas, etc.) en diferentes momentos del siglo XX. De modo particular, los artículos estudian las dinámicas y prácticas por las cuales estos agentes elaboraron un pensamiento crítico sobre la sociedad y la política argentina e implementaron diversas estrategias y prácticas intelectuales (individuales y colectivas) desde la universidad.

\footnotetext{
${ }^{1}$ La coordinación de este dossier forma parte del proyecto de I+D: "Universitarios en Argentina. Proyectos científicos y prácticas académicas, culturales y políticas, entre el siglo XX y los inicios del XXI", bajo la financiación del Departamento de Ciencias Sociales de la Universidad Nacional de Quilmes y la dirección de Osvaldo Graciano.

${ }^{2}$ Entre las obras colectivas se destaca especialmente los estudios reunidos en los siete tomos de la colección Dimensiones del Reformismo Universitario (Bacolla, Eujanián \& Mauro, 2018) y los libros de la Colección Reforma editados por la Universidad Nacional de Córdoba. A su vez, el portal de revistas AmericaLee del CeDInCi y el repositorio digital "A 100 años de la Reforma Universitaria", a cargo de CLACSO y la UNIPE, publicaron y digitalizaron revistas, compendios documentales y obras sobre el reformismo. Para análisis y balances historiográficos, ver (Buchbinder 2018; Bustelo 2018; Graciano 2019).
}

Esta obra está sujeta a la Licencia Reconocimiento-NoComercial-CompartirIgual 4.0 Internacional de Creative Commons. http://creativecommons.org/licenses/by-nc-sa/4.0/ 


\section{Luciana Carreño - Sebastián Gómez}

A partir del movimiento de la Reforma Universitaria, esa intervención de los agentes universitarios se expresó mediante una concepción de la actividad intelectual, que si bien emergía desde la universidad, se proyectaba en la vida pública a través de iniciativas de carácter sociocultural: edición de revistas, organización de ateneos, ciclos de conferencias y mítines, la militancia en el gremialismo estudiantil, entre otros espacios. ${ }^{3}$ Junto a la irrupción del reformismo, la reflexión por la dimensión pública de la universidad se enmarca, asimismo, dentro de una serie de estudios que han tomado por objeto a los intelectuales y agentes universitarios en su relación con el Estado y la política a lo largo del siglo XX.4

La perspectiva de análisis desarrollada en este dossier contribuye a identificar, en el largo plazo y a través de la reconstrucción situada en distintos escenarios (Córdoba, La Plata y Buenos Aires), una serie de trayectorias, proyectos culturales y políticos. A través de un enfoque relacional, las prácticas intelectuales y estudiantiles son analizadas a partir de procesos tales como las transformaciones en el sistema educativo; las lógicas de legitimación y constitución de las profesiones liberales; la emergencia de las ciencias sociales como disciplinas universitarias y profesionales; la circulación de agencias y agendas internacionales de investigación; la militancia política partidaria y extrapartidaria y la vinculación con centrales obreras y sindicales. A partir de esto, se comprende que las coyunturas de la historia política, si bien constituyen factores ineludibles, no pueden considerarse de modo exclusivo en la reflexión sobre las modalidades de intervención social y política de la universidad y de sus agentes.

Las movilizaciones y emprendimientos estudiantiles que se analizan en los dos primeros estudios del dossier se inscriben dentro de las nuevas prácticas políticas, sociales y culturales que se habilitan a partir de la Gran Guerra, la Revolución rusa y la experiencia democrática tras la introducción del sufragio obligatorio, secreto y universal-masculino. En ese marco, en ambos estudios es posible advertir las vinculaciones entre la acción pública estudiantil y las reconfiguraciones que, dentro y fuera de la universidad, se operaban en relación a las prácticas electorales, partidarias, asociativas y las tradiciones reformistas prexistentes. Pero al mismo tiempo, estas iniciativas de proyección pública emergían desde una determinada configuración del sistema universitario, y desde una formación y estructura matricular que, si bien continuaban caracterizándose por su función profesionalista

\footnotetext{
${ }^{3}$ Entre otros estudios esta perspectiva ha sido desarrollada por (Portantiero, 1978; Graciano, 2008; Biagini, 2012; Bustelo, 2015). Asimismo, existen varias investigaciones que abarcan la proyección política del movimiento estudiantil y del reformismo en distintos periodos del siglo XX. Al respecto véanse los dossiers (Millán \& Califa, 2018; Vega 2018).

${ }^{4}$ Los trabajos que abarcan estas dimensiones son varios. Junto con los citados sobre el papel político del reformismo, las vinculaciones entre intelectuales, universidad y política ha sido abordada, entre otros estudios, por (Sigal, 1991; Suasnabar, 2009; Terán, 2013; Petra, 2017). Otra línea de investigación se ha centrado específicamente en el papel que le cupo a la universidad y a sus agentes en la formación de cuadros técnicos, profesionales y políticos para las burocracias estatales ver (Buchbinder, 2010; Frederic, Graciano \& Soprano, 2010; Neiburg \& Plotkin, 2004; Di Liscia, \& Soprano, 2017).
} 


\section{Luciana Carreño - Sebastián Gómez}

y una inscripción social minoritaria y predominantemente masculina, experimentaban procesos de cambio.

En ese marco particular, el estudio de Ezequiel Grisendi analiza los contactos y las coyunturas que, luego de 1918, acercaron al movimiento estudiantil reformista con asociaciones y figuras del liberalismo progresista y los sectores obreros de la ciudad de Córdoba. Para lo cual el artículo toma por objeto las movilizaciones que estos sectores desplegaron en oposición a la estrategia de la Unión Popular Católica Argentina sobre la "cuestión social": la Gran Colecta Nacional. La investigación empírica que desarrolla el autor, a través de la prensa local y publicaciones periódicas del reformismo, logra llevarse a cabo desde una doble clave analítica e interpretativa. Por un lado, el autor atiende tanto a las disputas y complejidades al interior del espacio reformista como a las relaciones de los agentes universitarios con las fuerzas políticas del periodo. Por otro lado, desarrolla una reconstrucción en la cual las alianzas y estrategias de intervención pública que se aglutinaban en el frente anticatólico se analizan en relación a prácticas prexistentes de la cultura política local y a tradiciones del reformismo social que excedían la dimensión universitaria. Desde este enfoque relacional, el estudio de las movilizaciones y solidaridades que generó La Gran Colecta Nacional en la ciudad de Córdoba le permite al autor reflexionar sobre los límites de la opción reformista de representar una alternativa política para los sectores medios y trabajadores y sobre las modalidades concretas que adoptaron los líderes estudiantiles al intentar inscribir al reformismo dentro de un horizonte de reforma social.

En el artículo a cargo de Luciana Carreño se reconstruye la constitución de una red de revistas independientes de medicina que conectó a agrupaciones estudiantiles de las universidades nacionales de Buenos Aires y Córdoba, entre 1918 y 1926. A partir de este objeto, el estudio de las prácticas políticas de los estudiantes reformistas se analiza en articulación con los procesos de profesionalización de la carrera y el ejercicio de la medicina y con los cambios sociales que se operaban en la universidad. La interrelación de estas variables se examina, entre otros aspectos, a través la reformulación de ciertas modalidades de intervención pública (propias de las estrategias de la corporación médica) que realizan estas revistas desde una función crítica sobre las injusticias del orden social entonces vigente. Asimismo, estas vinculaciones se analizan a través de una prédica democratizadora $\mathrm{y}$ meritocrática, en la que se expresaba simultáneamente la socialización de conductas de compromiso político y social; nuevos preceptos de ética profesional entre los estudiantes y el proceso diversificación de la matrícula de cual formaban parte los editores y colaboradores de estas revistas.

Las contribuciones de Nayla Pis Diez y Sebastián Gómez se concentran en la trama política y universitaria de mediados del siglo XX. En el primer caso, la autora reconstruye la historia del movimiento estudiantil de la Universidad de La Plata (UNLP) en la década del 50. A fin de exponer las aporías, continuidades y transformaciones que experimentó dicho movimiento, Pis Diez distingue dos momentos: por un lado, los últimos años del segundo gobierno peronista; por otro, 


\section{Luciana Carreño - Sebastián Gómez}

los primeros años del posperonismo. En el estudio, propone un dinámico interjuego entre procesos históricos internacionales, nacionales y las especificidades propias del reformismo de la UNLP. De este modo, estudia de qué manera el reformismo platense fue dirimiendo, en una forma singular, los clivajes típicos de aquellos años: comunismo/anticomunismo, totalitarismo/democracia peronismo/antiperonismo. El artículo ofrece entonces una lectura procesual y de largo plazo sobre la politización del movimiento estudiantil radicado en la UNLP e identificado con la Reforma Universitaria. Lejos de una identidad fija e inmutable, la autora demuestra que el reformismo es una identidad histórica y, por tanto, ligada a los procesos e interpretaciones políticas de los propios agentes sobre las fuerzas sociales en pugna.

Cierra el dossier, la contribución de Sebastián Gómez abocada al análisis de la trayectoria juvenil de una figura gravitante en el campo de la educación: Juan Carlos Tedesco. Al abordar los años de formación del cientista de la educación, el artículo privilegia dos planos de análisis que se reclaman mutuamente: el político y el académico. En su reconstrucción, Gómez plantea características del campo político y académico de los años 50 y 60, por las que transitó Tedesco. Concretamente, analiza el derrotero del maestro normal por las tendencias izquierdistas del Partido Socialista y luego la agrupación troskista Política obrera, como así también por la carrera de Ciencias de la Educación de la Facultad de Filosofía y Letras de la Universidad de Buenos Aires (UBA). A lo largo del artículo, Gómez expone las diversas influencias políticas y académicas que jalonaron la formación de Tedesco y, más concretamente, su primer libro: Educación y sociedad, 1880-1900 (1970). Con todo, el artículo sugiere que la temprana formación de Tedesco permaneció modulada por un proceso híbrido entre dinámicas de modernización cultural y compromiso político.

Los artículos reunidos en el presente dossier contribuyen a reconstruir y reflexionar sobre las modalidades de intervención pública de agentes universitarios durante el siglo XX. En otras palabras, las intervenciones públicas promovidas por las identidades reformistas son analizadas en una clave histórica, demostrando la porosidad de tales identidades a los clivajes de los diferentes momentos del siglo XX. De este modo, el dossier promueve un análisis de la universidad, sus agentes y la propia opción reformista en sus múltiples intersecciones históricas con la vida societal.

Luciana Carreño Consejo Nacional de Investigaciones Científicas y Técnicas, Centro de Estudios en Historia, Cultura y Memoria, Departamento de Ciencias Sociales, Universidad Nacional de Quilmes lcarreno1@uvq.edu.ar 
Sebastián Gómez

Universidad de Buenos Aires;

Consejo Nacional de Investigaciones Científicas y Técnicas (Argentina)

sebastianjorgegomez@gmail.com

\section{Bibliografía}

Bacolla, N, Eujanián A. \& Mauro, D. (2018) (coords.). Dimensiones del Reformismo Universitario, (7 tomos). Rosario: Humanidades y Artes Ediciones.

Biagini, N. (2012). La contracultura juvenil. De la emancipación a los indignados, Buenos Aires, Capital Cultural.

Buchbinder (2010 [2005]): Historia de las Universidades Argentinas, Buenos Aires, Sudamericana.

Buchbinder, P. (2018) La Reforma Universitaria en vísperas de su centenario: notas sobre su historiografía. Boletín del Instituto de Historia Argentina y Americana "Dr. Emilio Ravignani”, Tercera serie (49), pp. 176-196.

Bustelo, N. (2015): La reforma universitaria desde sus grupos y revistas. Una reconstrucción de los proyectos y las disputas del movimiento estudiantil porteño de las primeras décadas del siglo XX (1914-1928), tesis de doctorado inédita, Facultad de Humanidades de Ciencias de la Educación, Universidad Nacional de La Plata, La Plata.

Bustelo, N. (2018). Un fantasma que recorrió América Latina: A 100 años de la Reforma Universitaria. Nueva Sociedad, (275), pp. 147-156.

Di Liscia, M. S \& Soprano, G. (2017). Burocracias estatales. Problemas, enfoques y estudios de caso en la Argentina (entre fines del siglo XIX y XX). Rosario: Prohistoria Ediciones y UNLPAM.

Frederic, S., Graciano, O. y Soprano, G. (2010) (coords.). El Estado argentino y las profesiones liberales, académicas y armadas, Rosario, Prohistoria Ediciones.

Graciano, 0. (2008): Entre la torre de marfil y el compromiso político. Intelectuales de izquierda en la Argentina, 1918-1955, Bernal, Universidad Nacional de Quilmes.

Graciano, O. (2019) Dossier. La Reforma Universitaria de 1918 en su Centenario. historiapolitica.com.

Disponible

en: 


\section{Luciana Carreño - Sebastián Gómez}

http://www.historiapolitica.com/dossiers/reforma-universitaria-de-1918-en-sucentenario/

Millán, M., \& Califa, J. S. (2018) (coords). Presentación del dossier: Tras las huellas de la Reforma Universitaria. Archivos De Historia Del Movimiento Obrero Y La Izquierda, (12), pp. 9-10. https://doi.org/10.46688/ahmoi.n12.62

Neiburg, F. \& Plotkin, M. (2004) (comp.). Intelectuales y expertos. La constitución del conocimiento social en la Argentina. Buenos Aires: Paidós.

Petra, A. (2017). Intelectuales y cultura comunista: itinerarios, problemas y debates en la Argentina de posguerra. Buenos Aires: Fondo de Cultura Económica.

Portantiero, J. C. (1978): Estudiantes y política en América Latina. El proceso de la Reforma Universitaria (1918-1938), México, Siglo XXI.

Sigal, S. (1991). Intelectuales y poder en la década del sesenta, Bs. As.: Ediciones Punto Sur.

Suasnábar, C. (2009). La Reforma Universitaria de Córdoba de 1918: una mirada histórica de la relación entre intelectuales, universidad y política en la Argentina. Práxis Educativa, 4(1), pp. 51-61.

Terán, 0. (2013). Nuestros años sesenta. La formación de la nueva izquierda intelectual argentina, 1956-1966. Buenos Aires: Siglo XXI.

Vega, N. (2018) (coord.). Presentación dossier El movimiento estudiantil argentino y latinoamericano historia, debates y perspectivas a 100 años de la Reforma Universitaria y 50 de la Revolución Mundial. Páginas, 10 (23), pp. 3-7. http://revistapaginas.unr.edu.ar/index.php/RevPaginas/index. 